University of South Carolina

Scholar Commons

8-13-2008

\title{
Structure of the Thioredoxin-like Domain of Yeast Glutaredoxin 3
}

Lydia M. Gibson

University of South Carolina - Columbia

Nin N. Dingra

University of South Carolina - Columbia

Caryn E. Outten

University of South Carolina - Columbia, outten@mailbox.sc.edu

Lukasz Lebioda

University of South Carolina - Columbia, lebioda@mailbox.sc.edu

Follow this and additional works at: https://scholarcommons.sc.edu/chem_facpub

Part of the Chemistry Commons

\section{Publication Info}

Published in Acta Crystallographica Section D: Biological Crystallography, Volume 64, Issue 9, 2008, pages 927-932.

(C) Acta Crystallographica Section D: Biological Crystallography 2008, International Union of Crystallography. accepted for inclusion in Faculty Publications by an authorized administrator of Scholar Commons. For more information, please contact digres@mailbox.sc.edu. 
Acta Crystallographica Section D

Biological Crystallography

ISSN 0907-4449

Lydia M. Gibson, ${ }^{a}$ Nin N. Dingra, ${ }^{a}$ Caryn E. Outten ${ }^{\mathrm{a}, \mathrm{b}_{*}}$ and Lukasz Lebioda $^{\mathrm{a}, \mathrm{b}_{*}}$

a Department of Chemistry and Biochemistry, University of South Carolina, Columbia, SC 29208, USA, and ${ }^{\mathbf{b} C e n t e r}$ for Colon Cancer Research, University of South Carolina, Columbia, SC 29208, USA

Correspondence e-mail: caryn.outten@mail.chem.sc.edu, lebioda@mail.chem.sc.edu

\section{Structure of the thioredoxin-like domain of yeast glutaredoxin 3}

Yeast glutaredoxin 3 (Grx3) is a cytosolic protein that regulates the activity of the iron-responsive transcriptional activator Aft1. This member of the monothiol glutaredoxin family contains a thioredoxin-like domain and a glutaredoxinlike domain, which both possess a monothiol active site. The crystal structure of the thioredoxin-like domain has been determined at $1.5 \AA$ resolution and represents the first published structure of this domain for the monothiol glutaredoxin family. The loop containing the signature motif WA $x x \mathrm{C}$ is partially disordered, indicating a greater degree of flexibility in this region compared with classical dithiol thioredoxins with a WCGPC active-site motif.

\section{Introduction}

Thioredoxins (Trxs) and glutaredoxins (Grxs) are small oxidoreductases (usually $9-15 \mathrm{kDa}$ ) that are found in all types of organisms. Both proteins are members of the thioredoxinfold superfamily and typically catalyze thiol-disulfide exchange reactions. The classical dithiol Trxs utilize a highly conserved WCGPC active-site motif to reduce disulfides in substrate proteins. The dithiol Grxs possess a CPYC activesite motif and primarily catalyze glutathionylation and deglutathionylation of target proteins (Berndt et al., 2008). A subfamily of Grxs were recently identified that are characterized by a conserved monothiol CGFS active-site motif (Herrero \& de la Torre-Ruiz, 2007). Saccharomyces cerevisiae has three Grx-like proteins that belong to this subclass: yGrx3, yGrx4 and yGrx5. yGrx5 contains a single Grx-like domain and is localized to the mitochondrial matrix, where it plays an ill-defined role in $\mathrm{Fe}-\mathrm{S}$ cluster biogenesis (RodriguezManzaneque et al., 2002). However, recent evidence from studies of yGrx5 homologues in chloroplasts suggest that this protein may act as a scaffold for $\mathrm{Fe}-\mathrm{S}$ cluster assembly and transfer (Bandyopadhyay et al., 2008). Homologues of yGrx5 with a single Grx-like domain are found in both prokaryotes and eukaryotes (Herrero \& de la Torre-Ruiz, 2007).

Yeast Grx3 and Grx4 are highly homologous proteins that possess both an N-terminal Trx-like domain and a C-terminal Grx-like domain (Herrero \& de la Torre-Ruiz, 2007). Both proteins are cytosolic and are required for iron-responsive inhibition of the transcriptional activator Aft1 (Kumanovics et al., 2008; Ojeda et al., 2006; Pujol-Carrion et al., 2006). The conserved cysteine in the CGFS active site located in the Grxlike domain of yGrx3 and yGrx4 is required for interaction with Aft 1 and regulation of its activity (Ojeda et al., 2006). The
Received 20 May 2008

Accepted 11 July 2008

PDB Reference: thioredoxinlike domain of yeast glutaredoxin $3,3 \mathrm{~d} 6 \mathrm{i}, \mathrm{r} 3 \mathrm{~d} 6 \mathrm{isf}$.
(C) 2008 International Union of Crystallography Printed in Singapore - all rights reserved 
Table 1

Crystallographic data and refinement statistics.

Values in parentheses are for the outer shell.

\begin{tabular}{|c|c|}
\hline X-ray source & APS 22ID (SER-CAT) \\
\hline Wavelength $(\AA)$ & 1.0000 \\
\hline No. of frames (high pass/low pass) & $400 / 360$ \\
\hline Oscillation range (high pass/low pass) $\left(^{\circ}\right)$ & $0.5 / 0.5$ \\
\hline $\begin{array}{l}\text { Crystal-to-detector distance } \\
\text { (high pass/low pass) (mm) }\end{array}$ & $200 / 300$ \\
\hline Temperature $(\mathrm{K})$ & 100 \\
\hline Space group & $P 2_{1}$ \\
\hline \multicolumn{2}{|l|}{ Unit-cell parameters } \\
\hline$a(\AA)$ & 47.91 \\
\hline$b(\AA)$ & 48.61 \\
\hline$c(\AA)$ & 55.29 \\
\hline$\beta\left(^{\circ}\right)$ & 116.8 \\
\hline Volume $\left(\AA^{3}\right)$ & 114980 \\
\hline Solvent content $(\%)$ & 46 \\
\hline Matthews coefficient $\left(\AA^{3} \mathrm{Da}^{-1}\right)$ & 2.3 \\
\hline Mosaicity $\left({ }^{\circ}\right)$ & 0.44 \\
\hline Resolution range $(\AA)$ & $32.1-1.50(1.55-1.50)$ \\
\hline Average redundancy & $3.5(1.9)$ \\
\hline Average $I / \sigma(I)$ & $18.7(2.6)$ \\
\hline Total no. of reflections & $121469(1744)$ \\
\hline No. of unique reflections & $35147(1646)$ \\
\hline Completeness (\%) & $95.9(69.1)$ \\
\hline Total linear $R_{\text {merge }}(\%)$ & $6.2(34.7)$ \\
\hline$R$ value $(R E F M A C 5)(\%)$ & $22.5(30.7)$ \\
\hline$R_{\text {free }}$ value $(R E F M A C 5)(\%)$ & $24.9(30.4)$ \\
\hline No. of water molecules & 145 \\
\hline Average $B$ factors (chain $A$ /chain $B)\left(\AA^{2}\right)$ & $17.6 / 17.4$ \\
\hline \multicolumn{2}{|l|}{ Ramachandran statistics } \\
\hline Residues in most favored regions (\%) & 92.7 \\
\hline Residues in additional allowed regions (\%) & 5.8 \\
\hline Residues in generously allowed regions (\%) & 1.6 \\
\hline Residues in disallowed regions (\%) & 0 \\
\hline
\end{tabular}

Trx-like domains have a WAxPC motif in place of the signature WCGPC active-site motif found in classical dithiol Trxs. Monothiol Grxs with this hybrid Trx-Grx domain architecture are only found in eukaryotes (Herrero \& de la Torre-Ruiz, 2007). In general, the Grx-like domains of this protein family are more highly conserved than the Trx-like domains (Isakov et al., 2000). The human homologue of yGrx3/4 (termed PICOT for PKC-interacting cousin of Trx) has an N-terminal Trx-like domain followed by two Grx-like domains (Isakov et al., 2000). The Trx-like domain specifically interacts with protein kinase $\mathrm{C}-\theta$ and is proposed to play a role in regulating the function of this kinase and its downstream targets (Witte $e t$ al., 2000).

Here, we present the crystal structure of the N-terminal Trxlike domain of yGrx 3 determined at $1.5 \AA$ resolution. This report represents the first published structural information for the Trx-like domain for any member of the monothiol glutaredoxin/PICOT family.

\section{Materials and methods}

\subsection{Protein expression and crystallization}

The yGrx3 open reading frame containing both the Trx-like and Grx-like domains but lacking the first 35 amino acids $(\Delta 1-$ 35) was cloned into pET21a and expressed in Escherichia coli
BL21 (DE3) (Novagen) as described previously (Kumanovics et al., 2008). Briefly, transformants were grown at $310 \mathrm{~K}$ until $\mathrm{OD}_{600}$ reached 0.6 and were induced with $1 \mathrm{~m} M$ isopropyl $\beta$-D1-thiogalactopyranoside. Cells were harvested $3 \mathrm{~h}$ after induction and stored at $193 \mathrm{~K}$. Cells were lysed using three freeze-thaw cycles and soluble protein was extracted with $50 \mathrm{~m} M$ Tris- $\mathrm{HCl} \mathrm{pH}$ 8.0, $5 \mathrm{~m} M$ DTT. The extract was loaded onto a DEAE anion-exchange column (GE Healthcare) equilibrated with $25 \mathrm{~m} M$ Bis-Tris $\mathrm{pH}$ 6.0, $5 \mathrm{mM}$ DTT and eluted with a salt gradient. The fractions containing yGrx3 were further purified by gel filtration using a Superdex 75 column (GE Healthcare) in $50 \mathrm{~m} M$ Tris- $\mathrm{HCl}$ pH 8.0, $150 \mathrm{~m} M$ $\mathrm{NaCl}, 5 \mathrm{~m} M$ DTT. The final pure protein was concentrated with the addition of $5 \%$ glycerol and stored at $193 \mathrm{~K}$. The typical yield was $\sim 30 \mathrm{mg}$ of protein per litre of cell culture. Protein purity was checked by SDS-PAGE and MALDI-TOF, indicating a single species corresponding to $28120 \mathrm{kDa}$ (the calculated molecular weight is $28130 \mathrm{Da}$ upon removal of Met36).

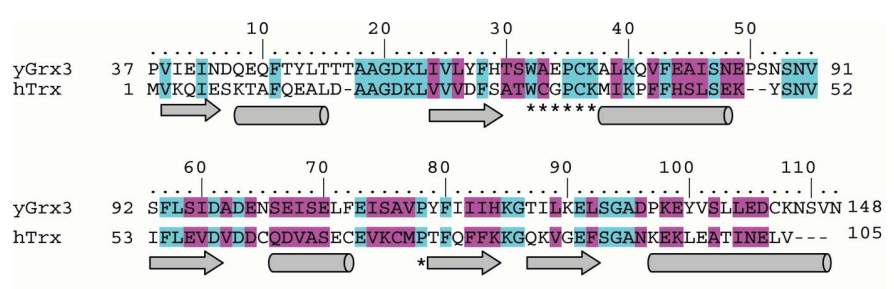

(a)

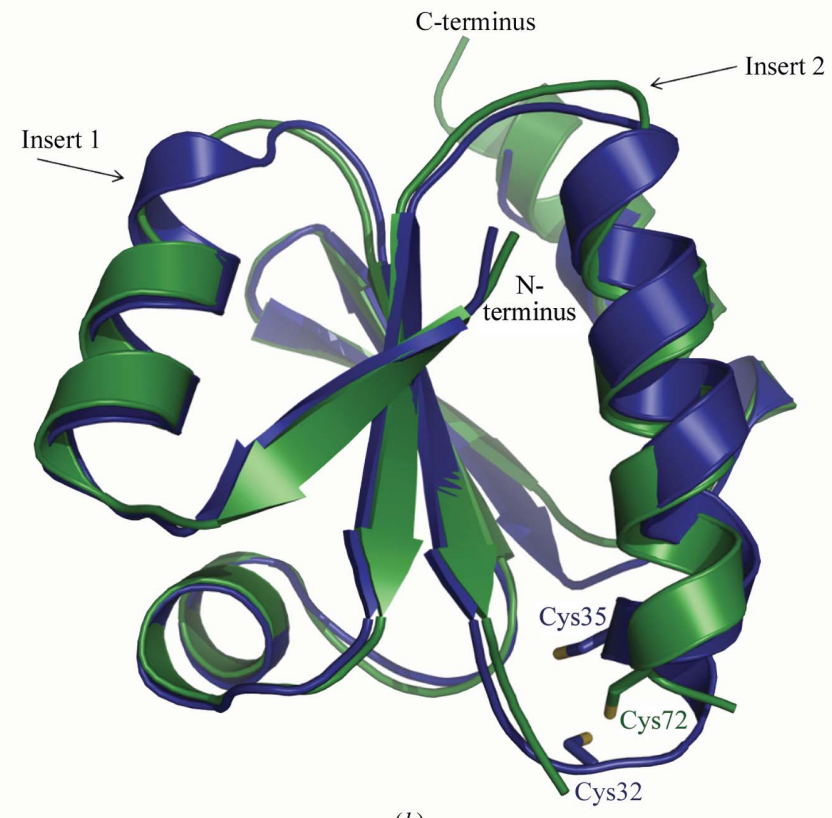

(b)

Figure 1

Comparison of yGrx3 Trx domain and hTrx. (a) Sequence alignment of the crystallized form of yGrx3 (amino acids 37-148) and the complete sequence of hTrx. The hTrx active-site motif and cis-Pro75 are indicated by asterisks. (b) Superposition of yGrx3 Trx domain (green) with Cys 72 in green atom type and hTrx (PDB code 1ert; blue) with Cys32 and Cys35 in blue atom type. The positions of the amino-acid inserts are marked by arrows. 
The final crystallization conditions were optimized from initial crystallization-condition leads obtained using Crystal Screen Lite from Hampton Research. Crystals of yGrx3 were grown under high-salt conditions by the hanging-drop method at $300 \mathrm{~K}$. Briefly, $2 \mu \mathrm{l}$ protein solution $\left(11 \mathrm{mg} \mathrm{ml}^{-1}\right.$ protein in $50 \mathrm{~m} M$ Tris $\mathrm{pH} 8.0$ and $5 \mathrm{~m} M$ DTT) was mixed with $2 \mu \mathrm{l}$ reservoir solution (0.1 $M$ MOPS pH 7.5, $20 \mathrm{~m} M \beta \mathrm{ME}$ and $68 \%$ saturated ammonium sulfate).

\subsection{Data collection and processing}

A yGrx3 crystal was transferred to cryosolvent $(60 \%$ ammonium sulfate, $20 \mathrm{~m} M \quad \beta \mathrm{ME}, 0.1 \mathrm{M}$ MOPS and $20 \%$ ethylene glycol) for a few seconds and subsequently flashfrozen in a liquid $\mathrm{N}_{2}$ vapor stream. Data were collected on the SER-CAT 22ID beamline at Argonne National Laboratory at
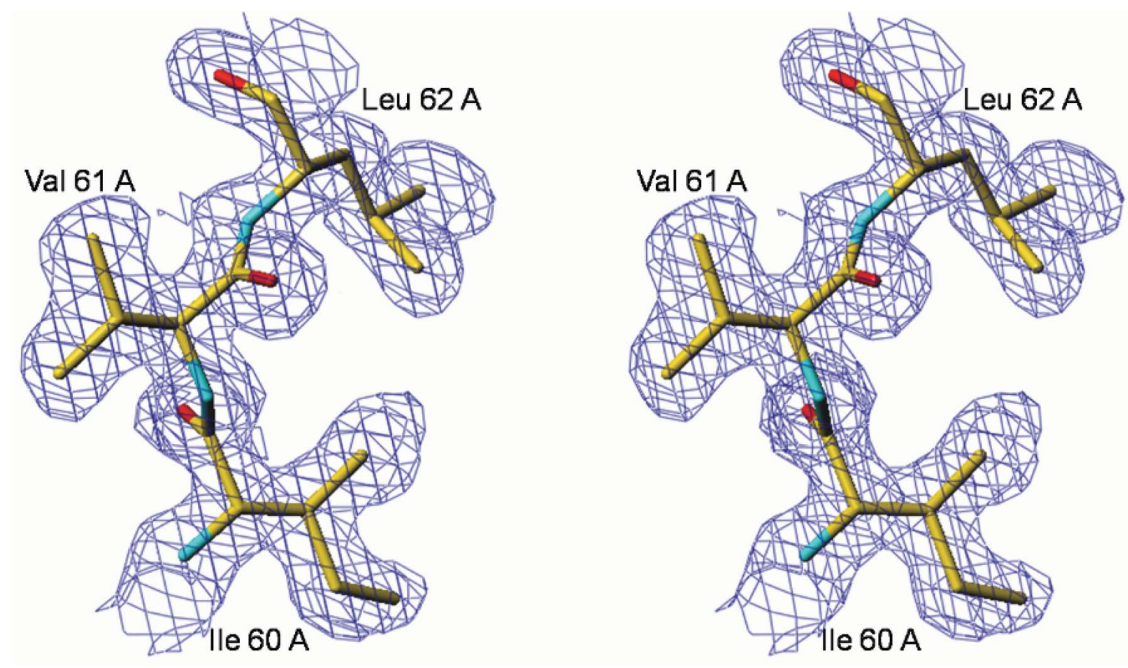

Figure 2

Representative electron-density map calculated with $2 F_{\mathrm{o}}-F_{\mathrm{c}}$ coefficients and contoured at the $1 \sigma$ level.
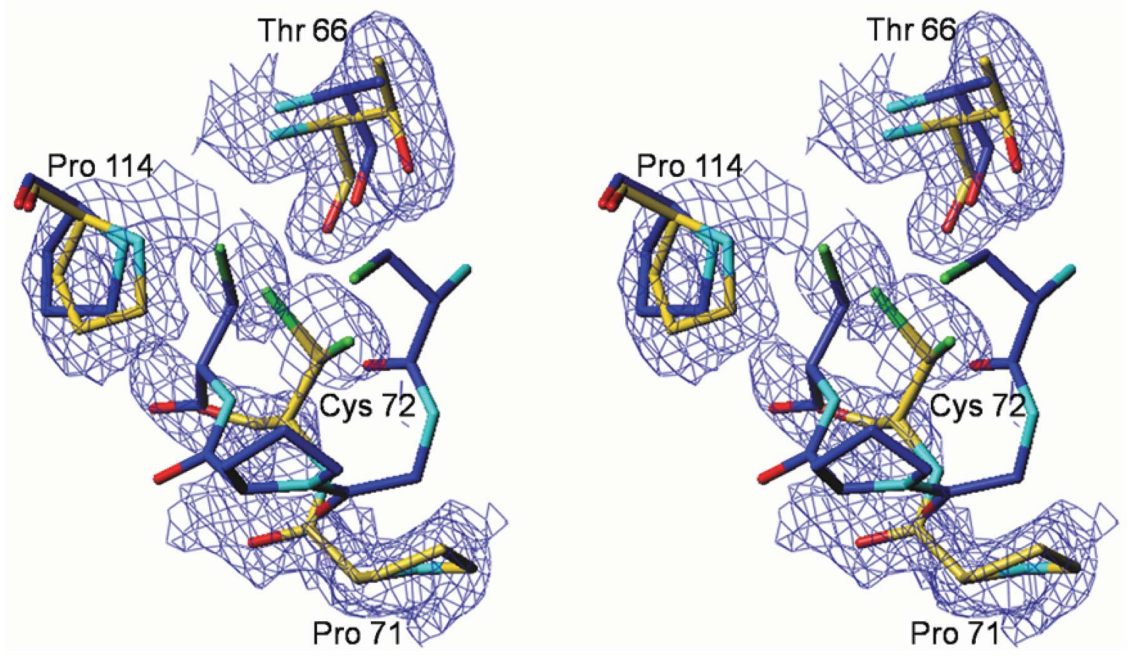

Figure 3

Stereoview of the putative active site in the Trx-like domain of yGrx3 subunit $A$ (yellow) superimposed with the human Trx active site (PDB code 1ert; blue). The OMIT map is contoured at $0.7 \sigma$. Labels are for $\mathrm{yGrx3}$. a $1.0000 \AA$ A wavelength in two passes: a first pass with a crystalto-detector distance of $200 \mathrm{~mm}$ and a second pass with a crystal-to-detector distance of $300 \mathrm{~mm}$, both using a $0.5^{\circ}$ oscillation range. The data were indexed and processed using the HKL-2000 software (Otwinowski \& Minor, 1997); processing parameters and statistics are summarized in Table 1.

\subsection{Structure determination and refinement}

The structure was solved by molecular replacement using $A M o R e$ (Navaza, 1994) from the CCP4 system (Collaborative Computational Project, Number 4, 1994) with the structure of the reduced form of human thioredoxin (hTrx) as the search model (PDB code 1ert; Weichsel et al., 1996). The amino-acid sequence of hTrx is $32 \%$ identical to that of the Trx-like domain of yGrx3 (see Fig. 1a). The initial model was rebuilt using $A R P / w A R P$ (Perrakis et al., 1999) from the $C C P 4$ suite, while subsequent interactive molecular-graphics and crystallographic refinements were carried out using TURBO FRODO (Roussel \& Cambillau, 1991) and CNS (Brünger et al., 1998), respectively. The final refinement was carried out with REFMAC5 (Murshudov et al., 1997). Superpositions were calculated using the $L S Q K A B$ program (Kabsch, 1976) from the $C C P 4$ suite. Figures were prepared using TURBO FRODO and PyMOL (DeLano, 2002).

\section{Results and discussion}

\subsection{Crystallization}

The recombinant yGrx3 protein was cloned from the second start site (encoding Met36) to the stop codon after determining that the first start site (encoding Met1) was not utilized in vivo (N. Dingra \& C. Outten, unpublished data). Furthermore, MALDITOF analysis indicated that Met36 is removed upon overexpression, as is typically found when the penultimate amino acid is relatively small (Pro37 in this case; Miller, 1975). Crystallization experiments were performed using this $28 \mathrm{kDa}$ protein that included both the Trx-like and Grx-like domains (amino acids 37-285). Two forms of crystals appeared after about four weeks under similar conditions. One form was tetragonal and diffracted poorly, while the other monoclinic form was superior and its characterization is the focus of this report. However, only the Trx-like domain is present in the monoclinic crystals, with two protomers per asymmetric unit and amino acids 37-148 identifiable in the electron density. The solvent content calculated with 
the two Trx-like domains in the asymmetric unit was $46 \%$, which is a typical value, while the solvent content calculated for two molecules of the Trx-Grx chimera protein was below $0 \%$. Therefore, we believe that the Grx-like domain was cleaved off during crystallization and the Trx-like domain was selectively crystallized. Dissociation of the Grx-like domain during crystallization suggests that the two domains do not maintain a strong interaction.

Subsequently, the Trx domain (amino acids 36-157) of yGrx3 was overexpressed (N. Dingra \& C. Outten, unpublished data). Its crystallization under similar conditions yielded both crystal forms. Tetragonal crystals appeared in $2 \mathrm{~d}$ and belonged to the $P 4$ space group with unit-cell parameters $a=76.42, c=37.16 \AA$. Despite good morphology and a size of $\sim 0.3 \mathrm{~mm}$, their diffraction only extended to $\sim 3.5 \AA$ resolution and thus this crystal form was not investigated further. Crystals of the monoclinic form appeared in $4 \mathrm{~d}$ (in contrast to the month needed to grow them from yGrx3). They were isomorphic to the crystals obtained from proteolysis of yGrx3 and had similar scattering power. Their structure was essentially the same and thus is not discussed here in detail.

\subsection{Overall structure}

Overall, both protomers have good electron density as shown in Fig. 2. A sequence alignment of the crystallized protein with hTrx is given in Fig. 1(a). The model contains residues $37-148$, but both subunits have a gap in the density in the putative active-site region (Fig. 1b). In subunit $A$ the gap is between residues 68 and 71 , while in subunit $B$ the gap is somewhat larger, between residues 68 and 74. This region is located on a surface-exposed loop. The two protomers are very similar: the r.m.s. distance between the $\mathrm{C}^{\alpha}$ positions is $0.58 \AA$ and they have similar corresponding temperature factors.

The yGrx3 Trx-like domain differs from hTrx by having one amino acid inserted after position 52 and two amino acids inserted after position 85 (Fig. 1a). Despite these differences, the overall fold of the yGrx3 Trx-like domain remains very similar to other known thioredoxins with the typical $\beta \alpha \beta \alpha \beta \alpha \beta \beta \alpha$ topology. The r.m.s. distance between the $\mathrm{C}^{\alpha}$ positions in the Trx-like domain $A$ and in hTrx (PDB code 1ert) is $1.14 \AA$.

\subsection{Putative active-site region}

The signature active-site WCGPC motif found in classical dithiol thioredoxins is different in the yGrx3 Trx-like domain and aligns with the sequence WAEPC in the putative active site. Density is present for the single Cys 72 in subunit $A$, which is moderately good for the main chain. It is likely that its side chain populates two conformations with some shift of the main chain involved. There is no density for $\mathrm{C}^{\beta}$, but there are two distinct peaks in electron density that are $2.7 \AA$ apart at positions that approximately correspond to two rotamers of the thiol. This distance is too short for a simple $\mathrm{SH}-\mathrm{H}_{2} \mathrm{O}$ contact, which should be $\sim 3.2 \AA$, but some combination of water/thiol disorder is clearly a possibility. Interestingly, the two positions of the partially disordered thiol group roughly correspond to the positions of the two cysteines found in dithiol thioredoxins (Fig. 3). The position of Pro71 is different
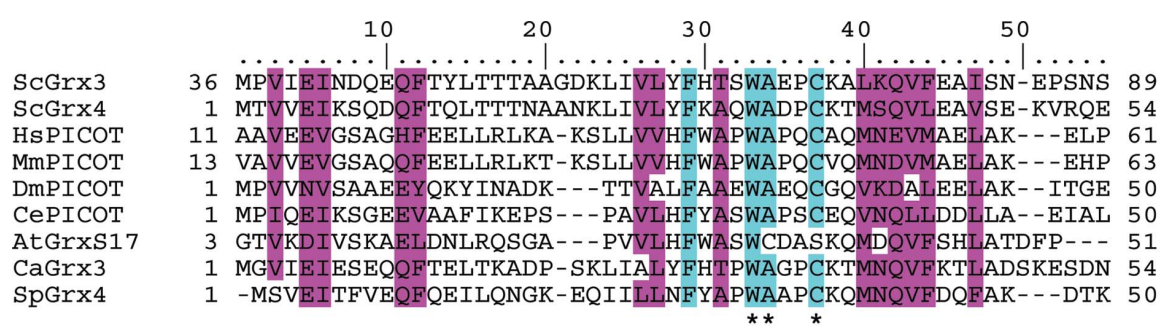

ScGrx3
ScGrx4
hsPICOT
mmPICOT
DmPICOT
CePICOT
AtGrxS17
CaGrx3
SpGrx4
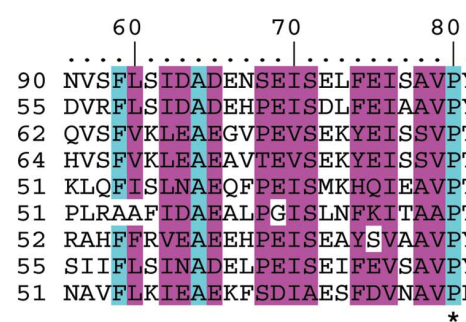

** *

\section{Figure 4}

Sequence alignment of the Trx-like domains of monothiol Grxs in several model organisms. Highly conserved residues are highlighted in blue and similar residues are shown in pink. The asterisks indicate the highly conserved residues in the putative active site. The sequences were aligned using ClustalW2 (Larkin et al., 2007). ScGrx3, Saccharomyces cerevisiae Grx3 (gi|633632); ScGrx4, S. cerevisiae Grx4 (gi|418432); HsPICOT, Homo sapiens PICOT (gi|6840947); MmPICOT, Mus musculus PICOT (gi|6840949); DmPICOT, Drosophila melanogaster PICOT (gi|19921242); CePICOT, Caenorhabditis elegans PICOT (gi|16604139); AtGrxS17, Arabidopsis thaliana GrxS17 (gi|22655350); CaGrx3, Candida albicans Grx3 (gi|46442105); SpGrx4, Schizosaccharomyces pombe $\mathrm{Grx} 4$ (gi|3650389).
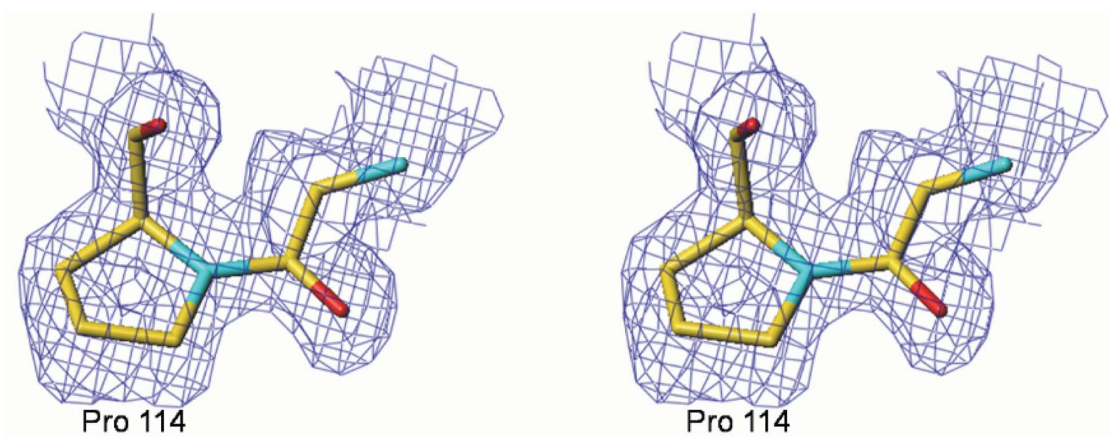

Figure 5

Stereoview of electron density for cis-Pro114 contoured at the $1 \sigma$ level. 
but well defined, while the residues corresponding to the rest of the signature motif (Ala69-Glu70) are disordered, indicating flexibility in this part of the structure. The electron density for Trp68 indicates that it is only slightly shifted in comparison to the corresponding residue in hTrx. There is a question whether the catalytic loop disorder might be related to the proteolysis of yGrx3, since this is a surface loop. This appeared to be possible when only the structure of the proteolytically cleaved Trx domain was available. However, the structure of the recombinant Trx domain also has the catalytic loop disordered. The recombinant Trx protein showed limited proteolysis after storage for about a month, but mass spectrometry showed that none of the major fragments corresponded to a cut in the disordered region.

The lack of conservation of active-site residues is not limited to the CxxC motif. Asp26 in $\mathrm{hTrx}$, which acts as a general acid-base catalyst and deprotonates the N-terminal cysteine in the WCGPC active site, is not present. The residues corresponding to Asp26 in monothiol Trx proteins are quite variable (histidine, tyrosine and leucine have been observed; Fig. 4), so its role is not likely to be significant. The lack of conservation of the active-site residues suggests that yGrx3 does not catalyze disulfide-bond reduction and that its regulatory function involves a different mechanism. However, it is possible that another cysteine from the Grx-like domain combines with Cys 72 to form a pair of thiols with reducing capabilities. If that is indeed the case, the flexibility of the region containing Cys 72 suggests that its thiol could penetrate substrate proteins more efficiently to reach a buried disulfide bond. For instance, human thymidylate synthase contains a highly reactive catalytic cysteine in the active-site cavity that readily forms a covalent bond with oxidized glutathione (L. M. Gibson, work to be published). To reduce such a buried bond, an oxidoreductase would have to penetrate the active-site cavity. This hypothesis can be perhaps verified when the structural relationship between the domains is established.

Both Trxs and Grxs typically contain a cis-proline located across from the conserved cysteine residues in the active site. This cis-proline (Pro114) is conserved in the Trx-like domain of yGrx3 with excellent electron density as shown in Fig. 5. In classical Trxs, the cis-proline is required for substrate binding and is proposed to position the mixed disulfide formed between Trx and its substrate for attack by the C-terminal cysteine (Qin et al., 1996). It is unclear whether Pro114 functions in this capacity in the Trx-like domain of yGrx3 since this domain lacks a second active-site cysteine. However, as mentioned above it is possible that a cysteine residue in the Grx-like domain interacts with the monothiol active site of the Trx-like domain to complete the disulfide-exchange reaction.

The presence of the conserved cis-proline has also been suggested to prevent metal binding by the redox-active cysteines in the active site of thioredoxin-fold proteins ( $\mathrm{Su}$ et al., 2007). However, several Grxs (including monothiol Grxs such as yGrx3) are able to bind a $2 \mathrm{Fe}-2 \mathrm{~S}$ cluster via active-site cysteines despite the presence of this conserved cis-proline (Bandyopadhyay et al., 2008; Johansson et al., 2007; Picciocchi et al., 2007). Therefore, an alternative possibility is that this domain functions in metal binding and/or metal transfer to the Grx-like domain. In fact, the high degree of flexibility in this putative active-site region is reminiscent of the $\mathrm{C} x x \mathrm{C}$ metalbinding loop in metallochaperones (Arnesano et al., 2002). Since the specific function of the Trx-like domain in yGrx3 has not been clearly defined, the significance of the conserved cisPro114 in both Fe-S cluster formation and substrate binding by yGrx3 requires further study.

This work was supported by NIH grant CA 76560 (LL) and NIH grant ES 13780 (CEO). Data were collected on the Southeast Regional Collaborative Access Team (SER-CAT) 22-ID beamline at the Advanced Photon Source, Argonne National Laboratory. Supporting institutions may be found at http://www.ser-cat.org/members.html. Use of the Advanced Photon Source was supported by the US Department of Energy, Office of Basic Energy Sciences under Contract No. W-31-109-Eng-38.

\section{References}

Arnesano, F., Banci, L., Bertini, I., Ciofi-Baffoni, S., Molteni, E., Huffman, D. L. \& O'Halloran, T. V. (2002). Genome Res. 12, 255-271.

Bandyopadhyay, S., Gama, F., Molina-Navarro, M. M., Gualberto, J. M., Claxton, R., Naik, S. G., Huynh, B. H., Herrero, E., Jacquot, J. P., Johnson, M. K. \& Rouhier, N. (2008). EMBO J. 27, 1122-1133.

Berndt, C., Lillig, C. H. \& Holmgren, A. (2008). Biochim. Biophys. Acta, 1783, 641-650.

Brünger, A. T., Adams, P. D., Clore, G. M., DeLano, W. L., Gros, P., Grosse-Kunstleve, R. W., Jiang, J.-S., Kuszewski, J., Nilges, M., Pannu, N. S., Read, R. J., Rice, L. M., Simonson, T. \& Warren, G. L. (1998). Acta Cryst. D54, 905-921.

Collaborative Computational Project, Number 4 (1994). Acta Cryst. D50, 760-763.

DeLano, W. L. (2002). The PyMOL Molecular Graphics System. http://www.pymol.org.

Herrero, E. \& de la Torre-Ruiz, M. A. (2007). Cell. Mol. Life Sci. 64, $1518-1530$.

Isakov, N., Witte, S. \& Altman, A. (2000). Trends Biochem. Sci. 25, 537-539.

Johansson, C., Kavanagh, K. L., Gileadi, O. \& Oppermann, U. (2007). J. Biol. Chem. 282, 3077-3082.

Kabsch, W. (1976). Acta Cryst. A32, 922-923.

Kumanovics, A., Chen, O. S., Li, L., Bagley, D., Adkins, E. M., Lin, H., Dingra, N. N., Outten, C. E., Keller, G., Winge, D., Ward, D. M. \& Kaplan, J. (2008). J. Biol. Chem. 283, 10276-10286.

Larkin, M. A., Blackshields, G., Brown, N. P., Chenna, R., McGettigan, P. A., McWilliam, H., Valentin, F., Wallace, I. M., Wilm, A., Lopez, R., Thompson, J. D., Gibson, T. J. \& Higgins, D. G. (2007). Bioinformatics, 23, 2947-2948.

Miller, C. G. (1975). Annu. Rev. Microbiol. 29, 485-504.

Murshudov, G. N., Vagin, A. A. \& Dodson, E. J. (1997). Acta Cryst. D53, 240-255.

Navaza, J. (1994). Acta Cryst. A50, 157-163.

Ojeda, L., Keller, G., Muhlenhoff, U., Rutherford, J. C., Lill, R. \& Winge, D. R. (2006). J. Biol. Chem. 281, 17661-17669.

Otwinowski, Z. \& Minor, W. (1997). Methods Enzymol. 276, 307-326.

Perrakis, A., Morris, R. \& Lamzin, V. S. (1999). Nature Struct. Biol. 6, 458-463.

Picciocchi, A., Saguez, C., Boussac, A., Cassier-Chauvat, C. \& Chauvat, F. (2007). Biochemistry, 46, 15018-15026.

Pujol-Carrion, N., Belli, G., Herrero, E., Nogues, A. \& de la TorreRuiz, M. A. (2006). J. Cell Sci. 119, 4554-4564. 


\section{research papers}

Qin, J., Clore, G. M., Kennedy, W. P., Kuszewski, J. \& Gronenborn, A. M. (1996). Structure, 4, 613-620.

Rodriguez-Manzaneque, M. T., Tamarit, J., Belli, G., Ros, J. \& Herrero, E. (2002). Mol. Biol. Cell, 13, 1109-1121.

Roussel, A. \& Cambillau, C. (1991). Silicon Graphics Geometry Partners Directory, p. 86. Mountain View, USA: Silicon Graphics.
Su, D., Berndt, C., Fomenko, D. E., Holmgren, A. \& Gladyshev, V. N. (2007). Biochemistry, 46, 6903-6910.

Weichsel, A., Gasdaska, J. R., Powis, G. \& Montfort, W. R. (1996). Structure, 4, 735-751.

Witte, S., Villalba, M., Bi, K., Liu, Y., Isakov, N. \& Altman, A. (2000). J. Biol. Chem. 275, 1902-1909. 